Supporting Information

\title{
Improvement of supercapacitor performance through enhanced interfacial interactions induced by sonication
}

\author{
Bhaskar J. Choudhury ${ }^{\mathrm{a}}$, Kuldeep Roy ${ }^{\mathrm{b}}$, Vijayanand S. Moholkara, b,* \\ ${ }^{a}$ Centre for Energy, Indian Institute of Technology Guwahati, Guwahati, Assam-781039, India \\ bDepartment of Chemical Engineering, Indian Institute of Technology Guwahati, Guwahati, Assam- \\ 781039, India \\ *Corresponding author: Fax: +91 361258 2291, E-mail: vmoholkar@iitg.ac.in
}


Table S1. Elemental compositions of GO and $\mathrm{rGO}$ from $\mathrm{CHN}$ analysis

\begin{tabular}{ccccc}
\hline Sample & C (wt. \%) & H (wt. \%) & N (wt. \%) & O (wt. \%) \\
\hline GO & 41.5 & 3.2 & 0 & 55.3 \\
rGO & 89.0 & 0.6 & 1.9 & 8.5 \\
\hline
\end{tabular}

Table S2. BET results of samples

\begin{tabular}{cccc}
\hline Sample & $S_{B E T}\left(\mathrm{~m}^{2} \mathrm{~g}^{-1}\right)$ & $V_{T}\left(\mathrm{~cm}^{3} \mathrm{~g}^{-1}\right)$ & $D_{A}(\mathrm{~nm})$ \\
\hline $\mathrm{rGO}$ & 555.20 & 0.49 & 3.57 \\
$\mathrm{Fe}_{3} \mathrm{O}_{4}$ & 104.83 & 0.27 & 9.19 \\
$\mathrm{Fe}_{3} \mathrm{O}_{4} / \mathrm{rGO}$ & 332.34 & 0.36 & 4.34 \\
$\mathrm{Fe}_{3} \mathrm{O}_{4} / \mathrm{rGO}$ & 227.18 & 0.30 & 5.30 \\
(without ultrasound) & & &
\end{tabular}

$S_{B E T}$ - BET surface area; $V_{T}$ - total pore volume; $D_{A}$ - average pore diameter

Table S3. Magnetic parameters of the of $\mathrm{Fe}_{3} \mathrm{O}_{4} \mathrm{NPs}$ and $\mathrm{Fe}_{3} \mathrm{O}_{4} / \mathrm{rGO}$ nanocomposite measured using vibrating sample magnetometer

\begin{tabular}{lrr}
\hline Parameter & $\mathrm{Fe}_{3} \mathrm{O}_{4}$ & $\mathrm{Fe}_{3} \mathrm{O}_{4} / \mathrm{rGO}$ \\
\hline Coercivity (Oe) & 22.37 & 16.57 \\
Magnetization $\left(\mathrm{emu} \mathrm{g}^{-1}\right)$ & 56.44 & 46.48 \\
Retentivity $\left(\mathrm{emu} \mathrm{g}^{-1}\right)$ & 1.61 & 0.80 \\
\hline
\end{tabular}


Table S4. Comparative evaluation of capacitance of symmetric solid-state supercapacitor devices

\begin{tabular}{|c|c|c|c|c|c|}
\hline Electrode materials & Electrolyte & $\begin{array}{l}\text { Voltage } \\
\text { window } \\
(\mathrm{V})\end{array}$ & $\begin{array}{l}\text { Specific } \\
\text { capacitance }\end{array}$ & $\begin{array}{l}\text { Capacitance } \\
\text { retention }\end{array}$ & Ref. \\
\hline PEDOT:PSS/MWCNT & $\mathrm{PVA} / \mathrm{KOH}$ & 1 & $\begin{array}{l}380 \mathrm{~F} \mathrm{~g}^{-1} \\
\text { at } 0.25 \mathrm{~A} \mathrm{~g}^{-1}\end{array}$ & $\begin{array}{l}90 \% \text { after } 1000 \\
\text { cycles }\end{array}$ & 58 \\
\hline $\mathrm{ZnS} / \mathrm{CNTs}$ & $\mathrm{PVA} / \mathrm{KOH}$ & 1 & $\begin{array}{l}159.6 \mathrm{~F} \mathrm{~g}^{-1} \\
\text { at } 1 \mathrm{~A} \mathrm{~g}^{-1}\end{array}$ & $\begin{array}{l}91.8 \% \text { after } \\
3000 \text { cycles }\end{array}$ & 55 \\
\hline $\begin{array}{l}\text { N-doped cotton-derived carbon } \\
\text { frameworks (NCCF)-rGO }\end{array}$ & $\mathrm{PVA} / \mathrm{KOH}$ & 1 & $\begin{array}{l}200 \mathrm{~F} \mathrm{~g} \mathrm{~g}^{-1} \\
\text { at } 0.1 \mathrm{~A} \mathrm{~g}^{-1}\end{array}$ & $\begin{array}{l}94 \% \text { after } \\
10000 \text { cycles }\end{array}$ & 53 \\
\hline $\begin{array}{l}\text { Polyaniline-coated carbon } \\
\text { nanofibers }\end{array}$ & $\mathrm{PVA} / \mathrm{H}_{2} \mathrm{SO}_{4}$ & 0.8 & $\begin{array}{l}201 \mathrm{~F} \mathrm{~g}^{-1} \\
\text { at } 0.25 \mathrm{~A} \mathrm{~g}^{-1}\end{array}$ & $\begin{array}{l}80 \% \text { after } 6000 \\
\text { cycles }\end{array}$ & 52 \\
\hline $\mathrm{ZnCo}_{2} \mathrm{O}_{4} / \mathrm{rGO}$ & $\mathrm{PVA} / \mathrm{KOH}$ & 0.4 & $\begin{array}{l}143 \mathrm{~F} \mathrm{~g}^{-1} \\
\text { at } 1 \mathrm{~A} \mathrm{~g} \mathrm{~g}^{-1}\end{array}$ & $\begin{array}{l}93.4 \% \text { after } \\
5000 \text { cycles }\end{array}$ & 51 \\
\hline Porous carbon & $\mathrm{PVA} / \mathrm{KOH}$ & 0.8 & $\begin{array}{l}81.3 \mathrm{~F} \mathrm{~g}^{-1} \\
\text { at } 0.5 \mathrm{~A} \mathrm{~g}^{-1}\end{array}$ & $\begin{array}{l}90.2 \% \text { after } \\
6000 \text { cycles }\end{array}$ & 57 \\
\hline $\mathrm{MWCNTs} / \mathrm{MnO}_{2}$ & $\mathrm{PVA} / \mathrm{Na}_{2} \mathrm{SO}_{4}$ & 1 & $\begin{array}{l}204 \mathrm{~F} \mathrm{~g}^{-1} \\
\text { at } 1 \mathrm{~mA} \mathrm{~cm}^{-2}\end{array}$ & $\begin{array}{l}80.36 \% \text { after } \\
2500 \text { cycles }\end{array}$ & 56 \\
\hline $\begin{array}{l}\text { Porous single-walled carbon } \\
\text { nanotube/poly-(3,4- } \\
\text { ethylenedioxythiophene) } \\
\text { (SWCNT/PEDOT) }\end{array}$ & $\mathrm{PVA} / \mathrm{H}_{3} \mathrm{PO}_{4}$ & 0.9 & $\begin{array}{l}53 \mathrm{Fg}^{-1} \\
\text { at } 1 \mathrm{Ag}^{-1}\end{array}$ & $\begin{array}{l}100 \% \text { after } \\
1000 \text { cycles }\end{array}$ & 54 \\
\hline $\mathrm{Fe}_{3} \mathrm{O}_{4} / \mathrm{rGO}$ & $\mathrm{PVA} / \mathrm{KOH}$ & 0.6 & $\begin{array}{l}169.2 \mathrm{~F} \mathrm{~g}^{-1} \\
\text { at } 1 \mathrm{~A} \mathrm{~g}^{-1}\end{array}$ & $\begin{array}{l}84.5 \% \text { after } \\
6000 \text { cycles }\end{array}$ & $\begin{array}{l}\text { This } \\
\text { work }\end{array}$ \\
\hline
\end{tabular}


Table S5. Comparison of electrochemical performance $\mathrm{Fe}_{3} \mathrm{O}_{4} / \mathrm{rGO}$ electrodes with recently reported iron oxide carbon-based electrodes

\begin{tabular}{|c|c|c|c|c|c|}
\hline Materials & Method & Electrolyte & Capacitance & $\begin{array}{l}\text { Capacitance } \\
\text { retention }\end{array}$ & Ref. \\
\hline $\begin{array}{l}\mathrm{Fe}_{3} \mathrm{O}_{4} \text {-carbon } \\
\text { nanosheets }\end{array}$ & Solvothermal & $1 \mathrm{M} \mathrm{Na}_{2} \mathrm{SO}_{3}$ & $\begin{array}{l}163.4 \mathrm{~F} \mathrm{~g}^{-1} \text { at } \\
1 \mathrm{~A} \mathrm{~g}^{-1}\end{array}$ & $\begin{array}{l}93 \% \text { after } 5000 \\
\text { cycles }\end{array}$ & 59 \\
\hline $\begin{array}{l}\text { Carbon } \\
\text { nanotube/cubic } \\
\mathrm{Fe}_{3} \mathrm{O}_{4}\end{array}$ & Hydrothermal & $6 \mathrm{M} \mathrm{KOH}$ & $\begin{array}{l}117.2 \mathrm{~F} \mathrm{~g} \mathrm{~g}^{-1} \text { at } \\
10 \mathrm{~mA} \mathrm{~cm}^{-2}\end{array}$ & $\begin{array}{l}91 \% \text { after } 500 \\
\text { cycles at } 10 \mathrm{~mA} \\
\mathrm{~cm}^{-2}\end{array}$ & 60 \\
\hline $\mathrm{FeOx} / \mathrm{CNF}$ & Electrospinning & $1 \mathrm{M} \mathrm{Na}_{2} \mathrm{SO}_{4}$ & $\begin{array}{l}436 \mathrm{~F} \cdot \mathrm{g}^{-1} \text { at } 1 \\
\mathrm{~A} \cdot \mathrm{g}^{-1}\end{array}$ & $\begin{array}{l}89 \% \text { after } 5000 \\
\text { cycles at } 1 \mathrm{~A} \cdot \mathrm{g}^{-1}\end{array}$ & 61 \\
\hline $\mathrm{Fe}_{3} \mathrm{O}_{4} /$ graphene & $\begin{array}{l}\text { Hydrothermal } \\
\text { method }\end{array}$ & $1 \mathrm{M} \mathrm{KOH}$ & $\begin{array}{l}661 \mathrm{~F} \mathrm{~g}^{-1} \text { at } \\
1 \mathrm{~A} \cdot \mathrm{g}^{-1}\end{array}$ & - & 62 \\
\hline $\mathrm{Fe}_{3} \mathrm{O}_{4} /$ graphene & Three step method & $1 \mathrm{M} \mathrm{KOH}$ & $\begin{array}{l}368 \mathrm{~F} \mathrm{~g}^{-1} \text { at } \\
1 \mathrm{~A} \mathrm{~g}^{-1}\end{array}$ & - & 63 \\
\hline $\begin{array}{l}\mathrm{Fe}_{3} \mathrm{O}_{4} / \text { reduced } \\
\text { graphene oxide }\end{array}$ & $\begin{array}{l}\text { Solvothermal } \\
\text { process }\end{array}$ & $1 \mathrm{M} \mathrm{KOH}$ & $\begin{array}{l}480 \mathrm{~F} \mathrm{~g}^{-1} \text { at } \\
5 \mathrm{~A} \mathrm{~g}^{-1}\end{array}$ & - & 30 \\
\hline $\begin{array}{l}\mathrm{Fe}_{3} \mathrm{O}_{4} / \text { reduced } \\
\text { graphene oxide }\end{array}$ & $\begin{array}{l}\text { Hydrolysis and } \\
\text { annealing process }\end{array}$ & $6 \mathrm{M} \mathrm{KOH}$ & $\begin{array}{l}350.6 \mathrm{~F} \mathrm{~g}^{-1} \text { at } \\
1 \mathrm{mV} \mathrm{s}^{-1}\end{array}$ & - & 64 \\
\hline $\begin{array}{l}\mathrm{Fe}_{3} \mathrm{O}_{4} / \text { Nitrogen-Doped } \\
\text { Carbon }\end{array}$ & $\begin{array}{l}\text { Reflux and } \\
\text { thermal expansion }\end{array}$ & $3 \mathrm{M} \mathrm{KOH}$ & $\begin{array}{l}522.7 \mathrm{~F} \mathrm{~g}^{-1} \text { at } \\
0.5 \mathrm{~A} \mathrm{~g}^{-1}\end{array}$ & $\begin{array}{l}91.9 \% \text { after } 5000 \\
\text { cycles } 2 \mathrm{~A} \mathrm{~g}^{-1}\end{array}$ & 65 \\
\hline $\begin{array}{l}\mathrm{Mn}_{3} \mathrm{O}_{4^{-}} \\
\mathrm{Fe}_{2} \mathrm{O}_{3} / \mathrm{Fe}_{3} \mathrm{O}_{4} @ \mathrm{rGO}\end{array}$ & Microwave & $1 \mathrm{M} \mathrm{KOH}$ & $\begin{array}{l}590.7 \mathrm{~F} / \mathrm{g} \text { at } \\
5 \mathrm{mV} \mathrm{s}^{-1}\end{array}$ & $\begin{array}{l}64.5 \% \text { after } 1000 \\
\text { cycles at } 50 \mathrm{mV} \mathrm{s}^{-1}\end{array}$ & 66 \\
\hline $\mathrm{Fe}_{3} \mathrm{O}_{4} / \mathrm{rGO}$ & $\begin{array}{l}\text { Ultrasound- } \\
\text { assisted method }\end{array}$ & $\mathrm{PVA} / \mathrm{KOH}$ & $\begin{array}{l}\# 676.8 \mathrm{~F} \mathrm{~g}^{-1} \text { at } \\
1 \mathrm{~A} \mathrm{~g}^{-1}\end{array}$ & $\begin{array}{l}84.5 \% \text { after } 6000 \\
\text { cycles }\end{array}$ & $\begin{array}{l}\text { This } \\
\text { work }\end{array}$ \\
\hline
\end{tabular}

${ }^{\#}$ Gravimetric capacitance $\left(\mathrm{C}_{\mathrm{g}}\right)$ of active material can be expressed as: $\mathrm{C}_{\mathrm{g}}=4 \mathrm{C}_{\mathrm{s}}$ where, $\mathrm{C}_{\mathrm{s}}=$ gravemetric specific capacitance of symmetric supercapacitors ${ }^{45}$ 


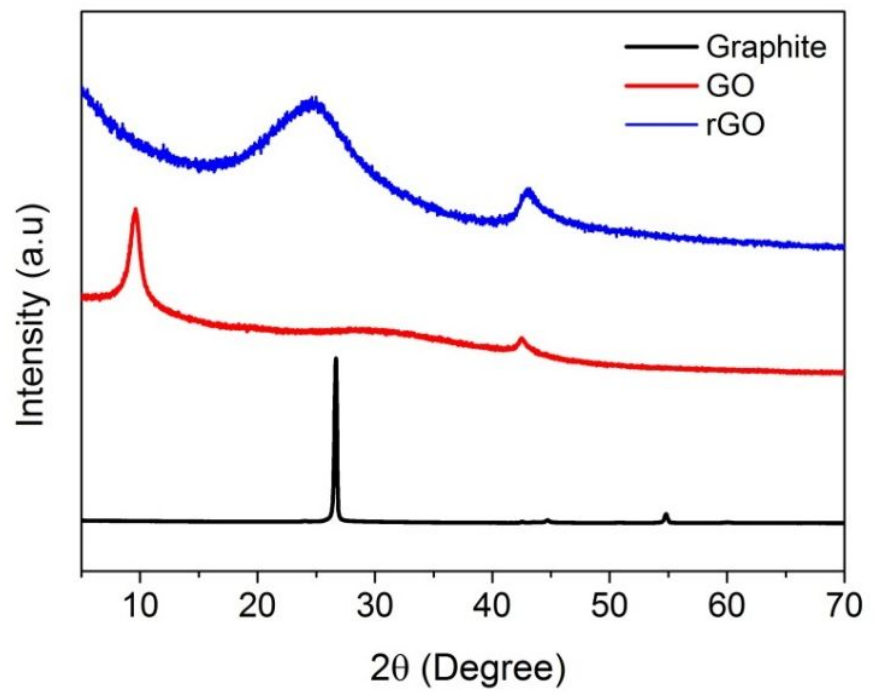

Figure S1. XRD plot of graphite, GO and rGO

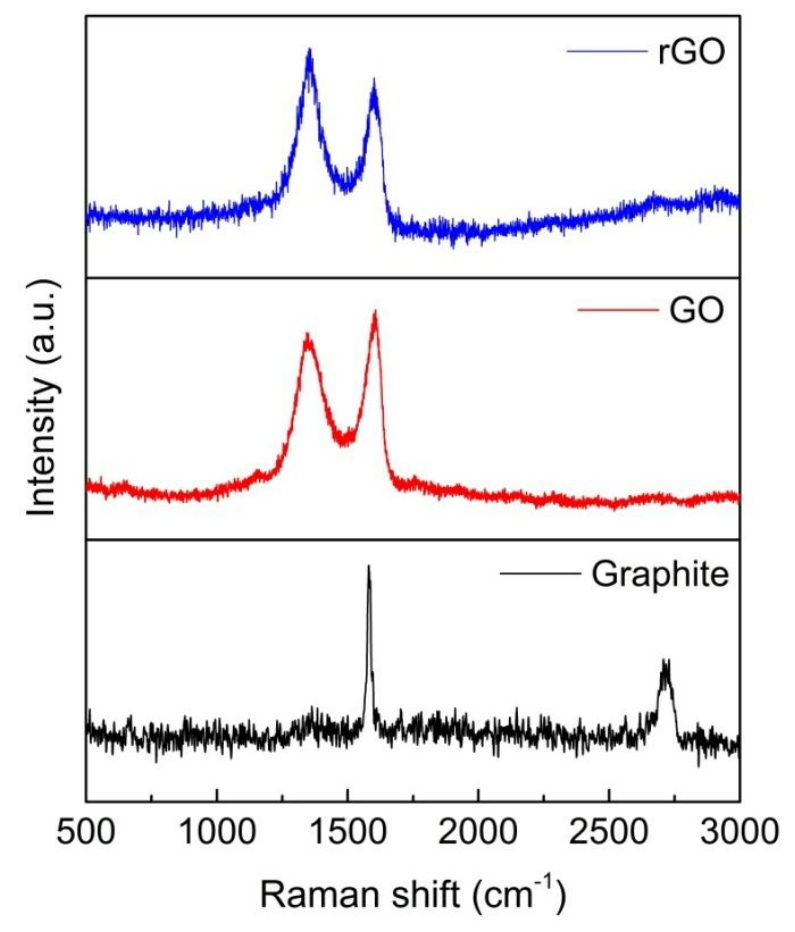

Figure S2. Raman spectra of graphite, GO and rGO 


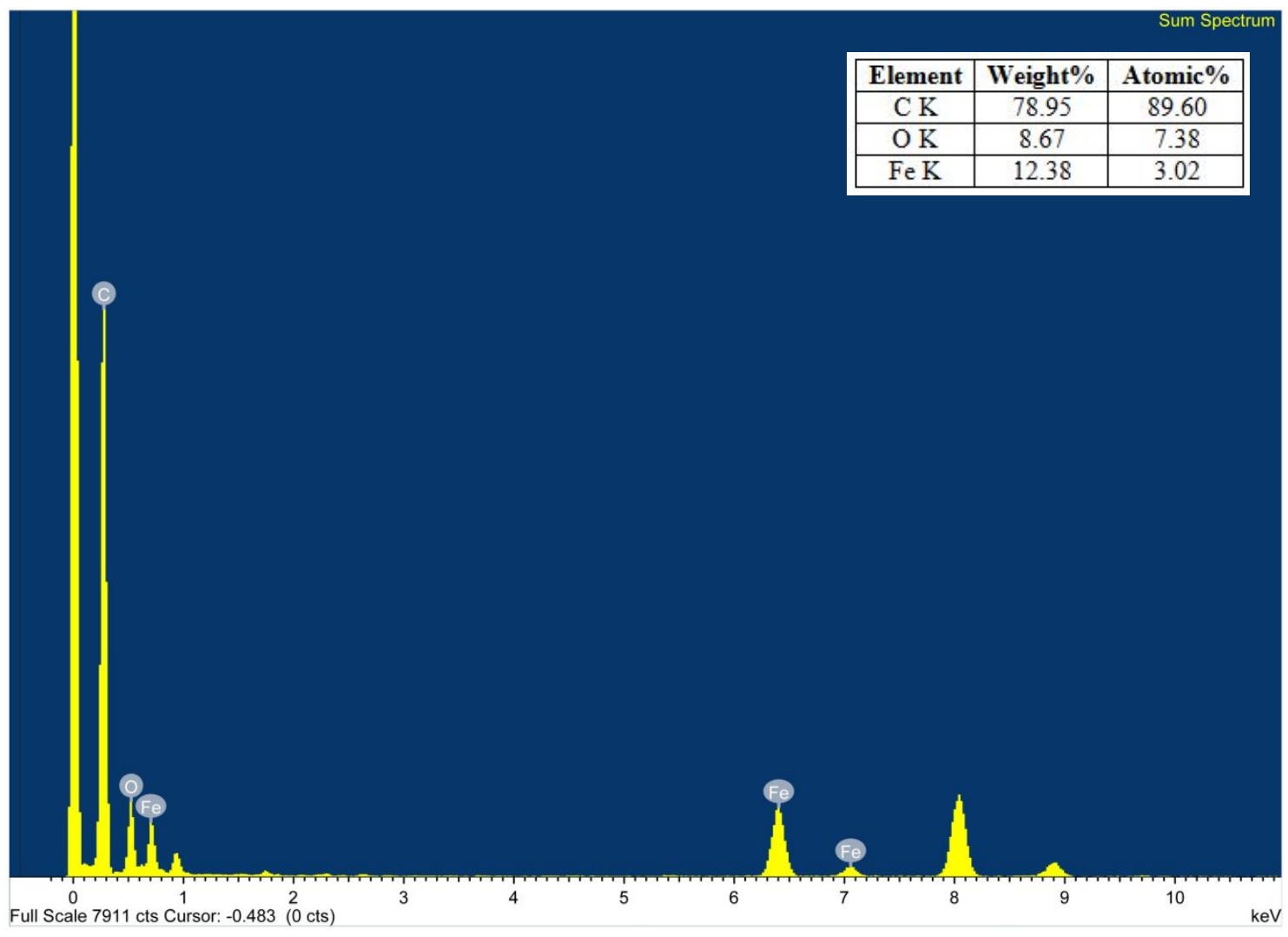

Figure S3. EDS spectrum of $\mathrm{Fe}_{3} \mathrm{O}_{4} / \mathrm{rGO}$ nanocomposite

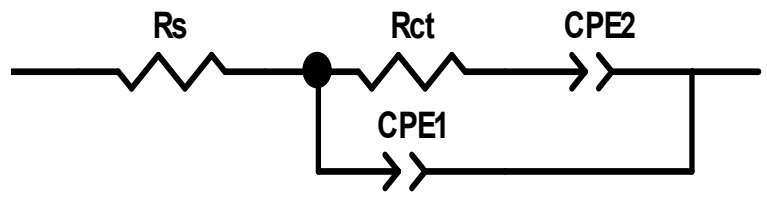

Figure S4. Equivalent circuit of ASSCs 


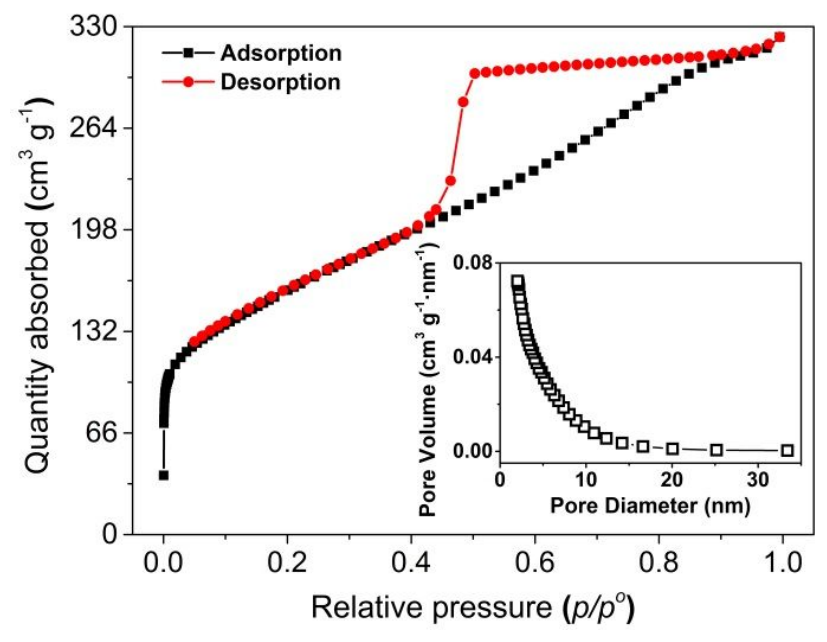

(a)

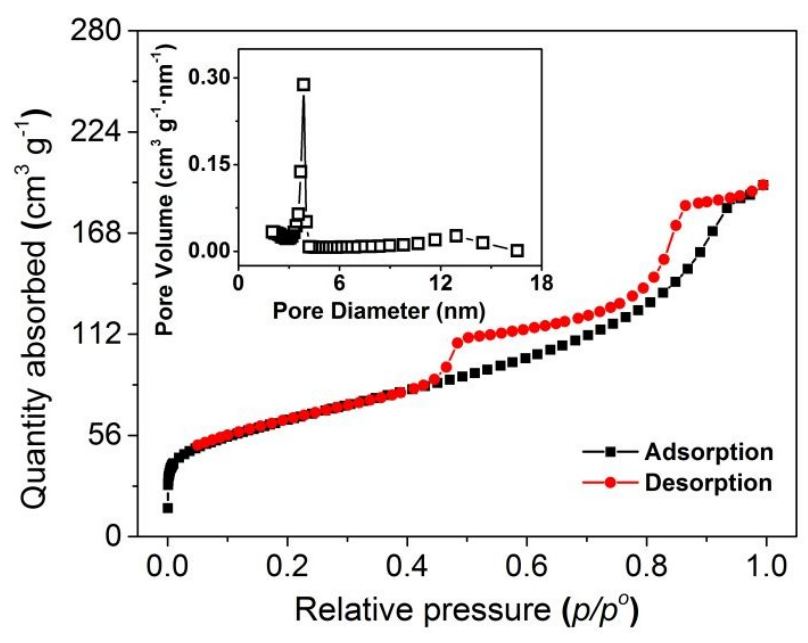

(b)

Figure S5. (a) Nitrogen adsorption-desorption isotherms (inset: BJH pore size distribution) of rGO (b) nitrogen adsorption-desorption isotherms (inset: $\mathrm{BJH}$ pore size distribution) of $\mathrm{Fe}_{3} \mathrm{O}_{4} / \mathrm{rGO}$ nanocomposites synthesized without ultrasound
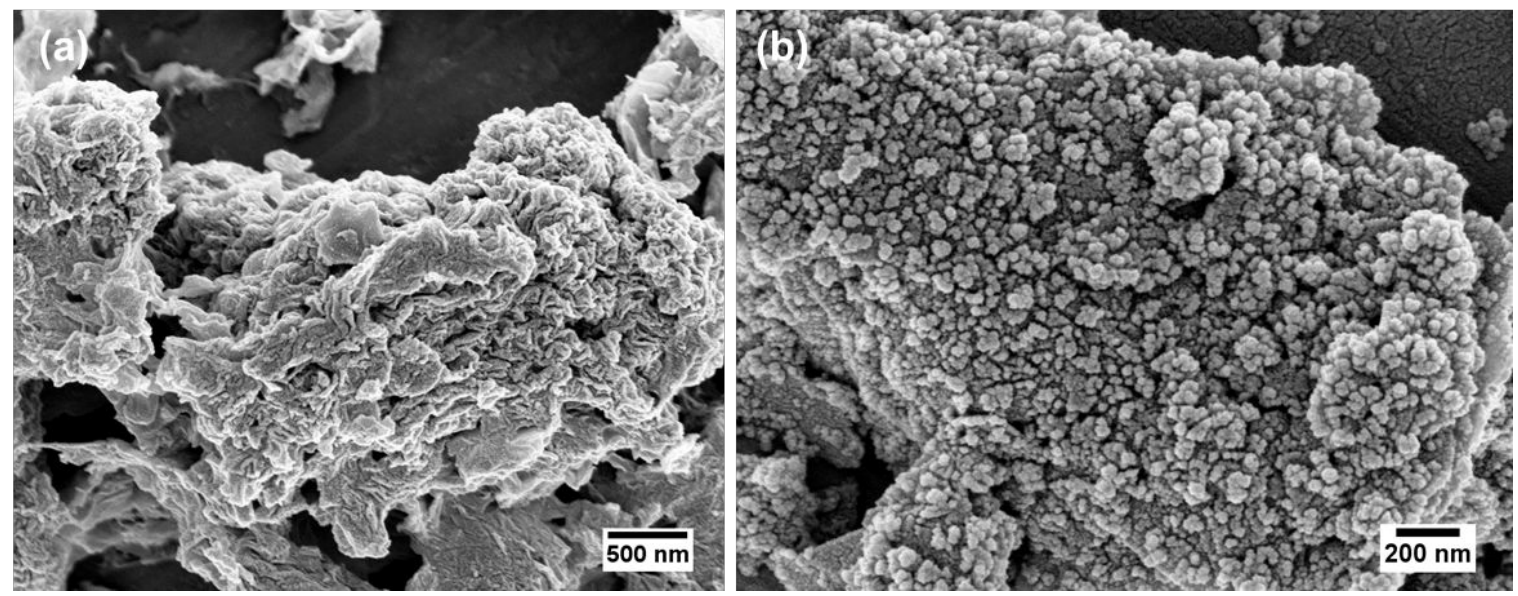

Figure S6. High magnification FE-SEM images of (a) $\mathrm{rGO}$, and (b) $\mathrm{Fe}_{3} \mathrm{O}_{4} / \mathrm{rGO}$ nanocomposite 


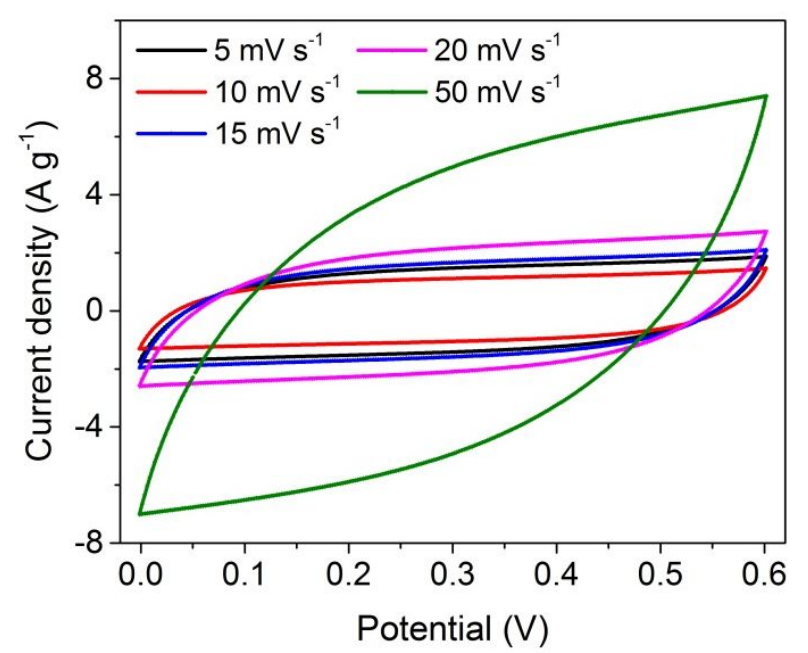

(a)

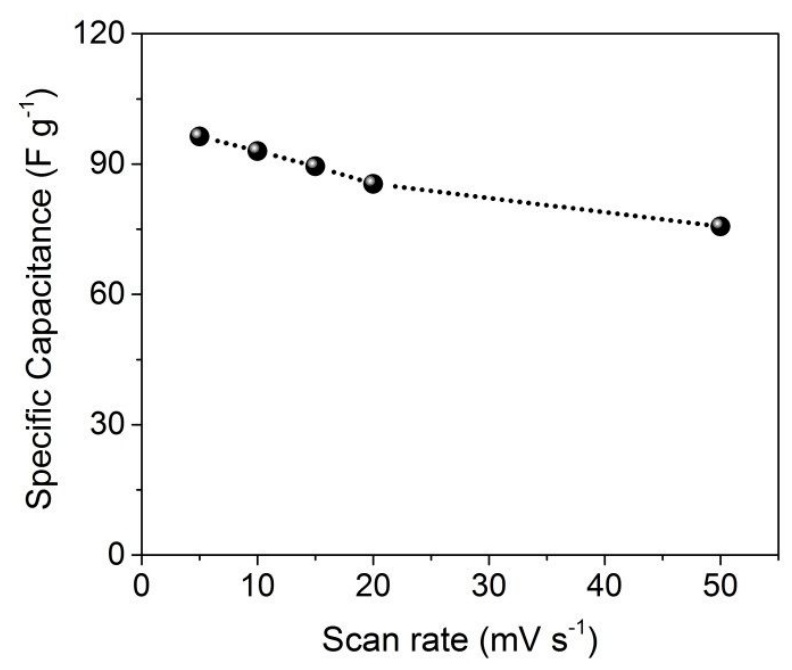

(c)

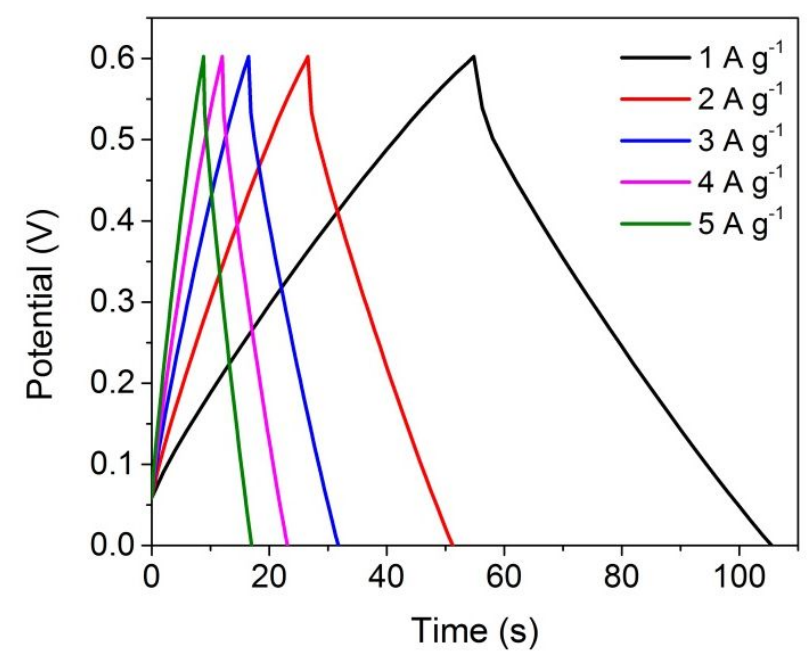

(b)

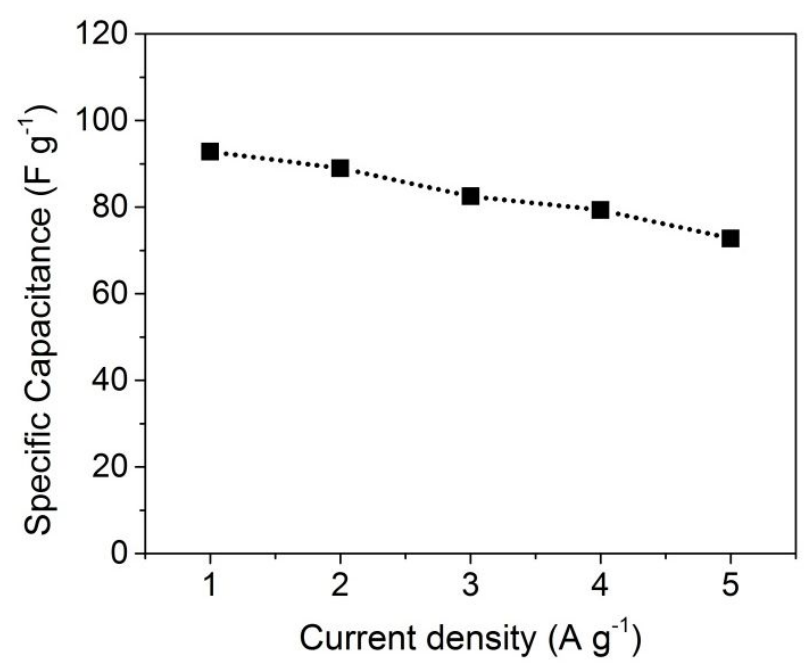

(d)

Figure S7. (a) Cyclic voltammograms, (b) galvanostatic charge-discharge curves, (c) specific capacitance vs. scan rate, (d) specific capacitance vs. current density of rGO ASSC 


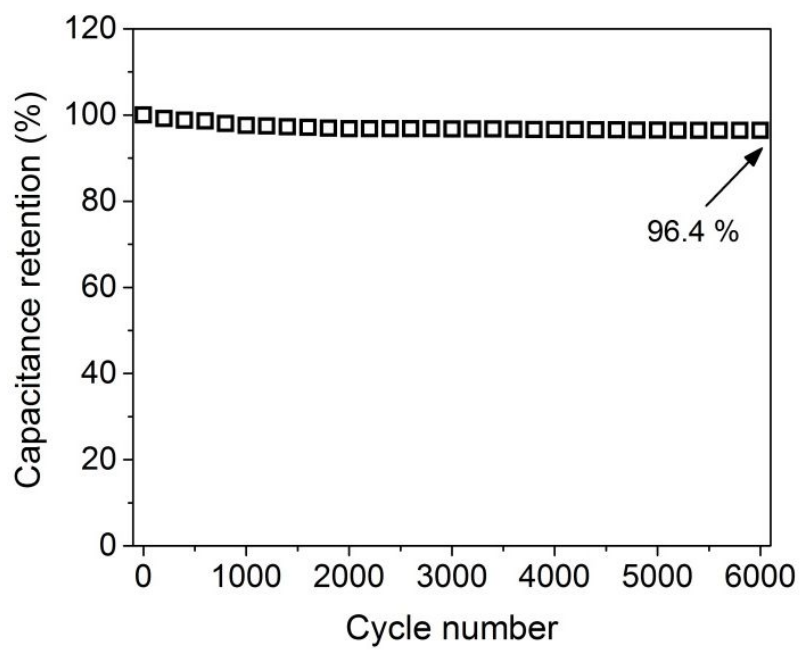

(a)

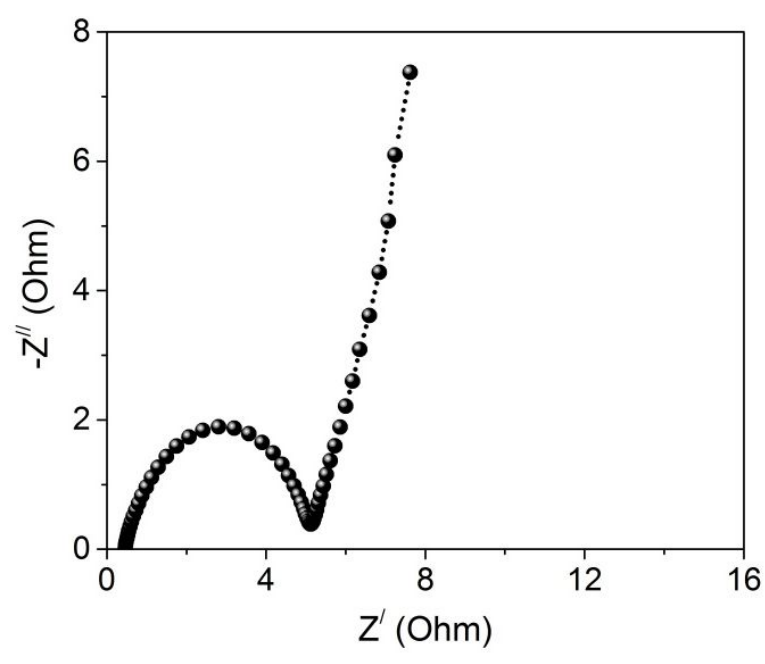

(b)

Figure S8. (a) Cyclic performance at $5 \mathrm{Ag}^{-1}$ and (b) Nyquist plot of rGO ASSC
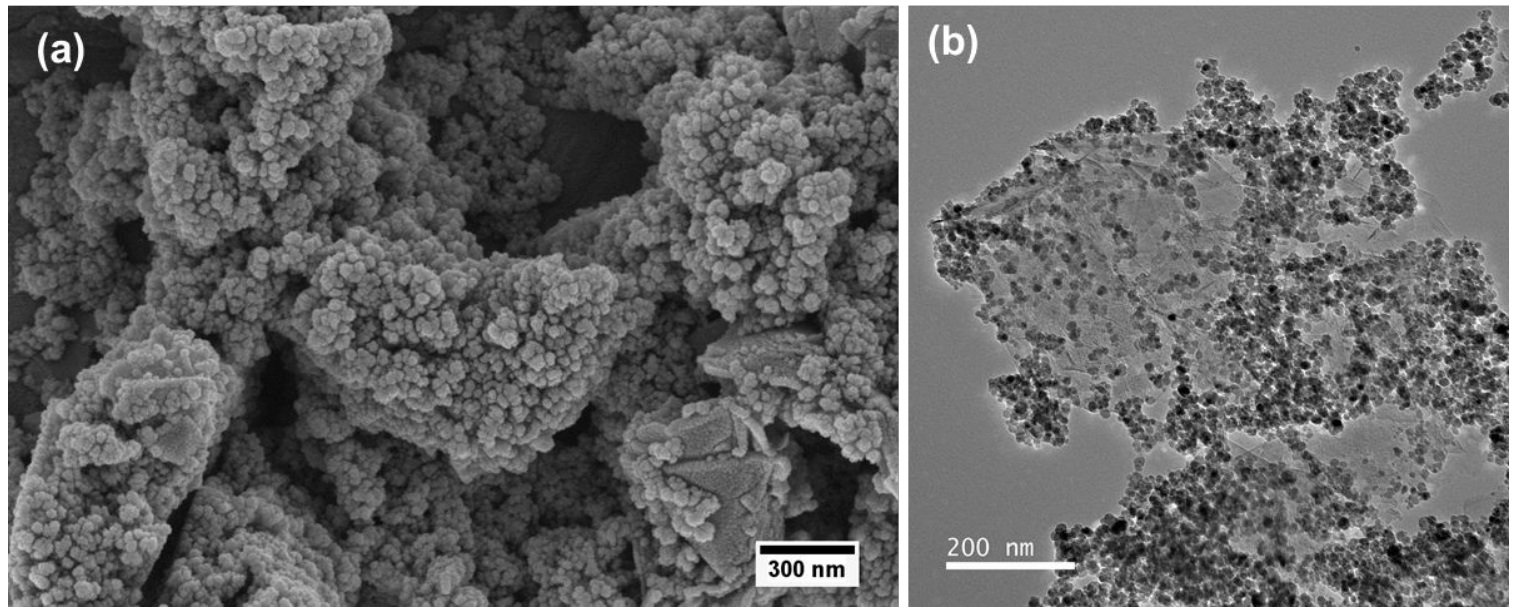

Figure S9. (a) FE-SEM and (b) TEM images $\mathrm{Fe}_{3} \mathrm{O}_{4} / \mathrm{rGO}$ nanocomposite synthesized without ultrasound 


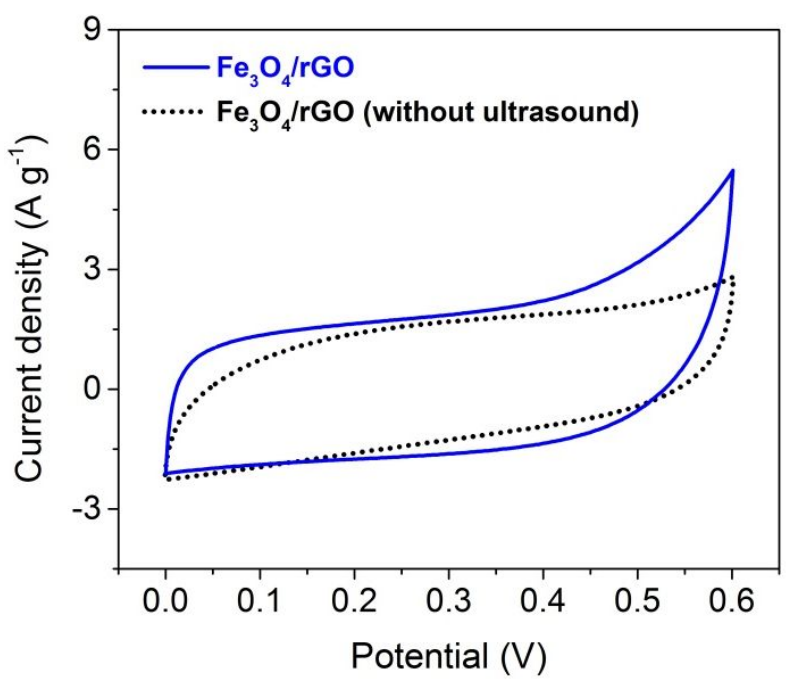

(a)

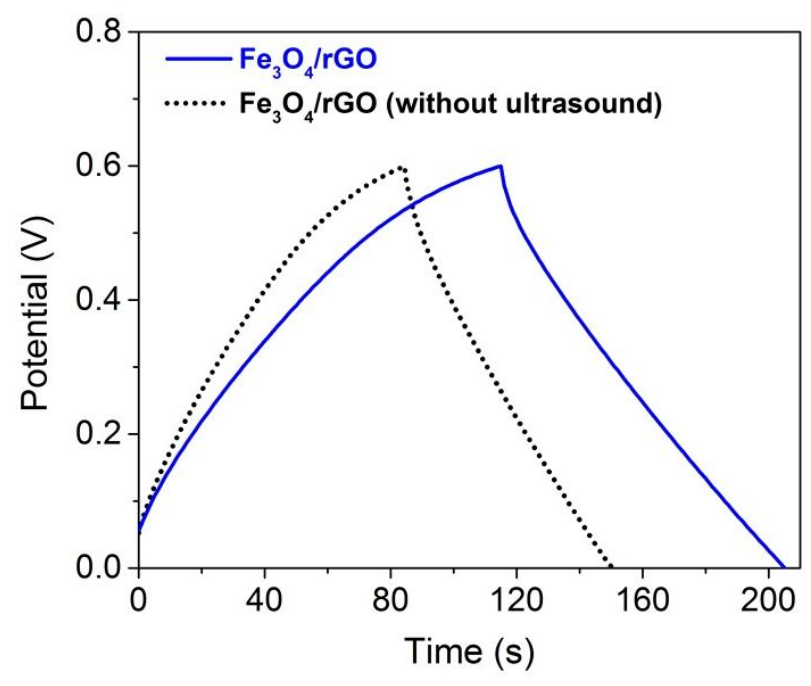

(b)

Figure S10. (a) Comparison of cyclic voltammograms at $10 \mathrm{mV} \mathrm{s}^{-1}$ (b) charge-discharge curves at 1 $\mathrm{A} \mathrm{g}^{-1}$ for $\mathrm{ASSCs}$ fabricated with $\mathrm{Fe}_{3} \mathrm{O}_{4} / \mathrm{rGO}$ nanocomposites synthesized using both ultrasoundassisted method and without ultrasound 